\title{
Multi-Objective Optimization Approach based on Deterministic and Metaheuristic Techniques to Resource Management in Health Crisis Scenarios under Uncertainty
}

Luis Germán Hernández-Pérez ( 0500305 d@umich.mx )

Universidad Michoacana de San Nicolás de Hidalgo

José María Ponce-Ortega

Universidad Michoacana de San Nicolás de Hidalgo

\section{Method Article}

Keywords: Deterministic optimization strategy, Mathematical model formulation, GAMS, MS Excel, SARSCoV-2, COVID-19

Posted Date: September 29th, 2020

DOI: https://doi.org/10.21203/rs.3.rs-83752/v1

License: (9) This work is licensed under a Creative Commons Attribution 4.0 International License.

Read Full License 


\title{
Multi-Objective Optimization Approach based on Deterministic and Metaheuristic
}

\section{Techniques to Resource Management in \\ Health Crisis Scenarios under Uncertainty}

\author{
Luis G. Hernández-Pérez \& José M. Ponce-Ortega*
}

Chemical Engineering Department, Universidad Michoacana de San Nicolás de Hidalgo, Francisco J. Mujica S/N, Edificio V1, Ciudad Universitaria, 58060, Morelia, Mich., México

* Corresponding author: J.M. Ponce-Ortega

E-mail: jmponce@umich.mx;

Tel. +52 4433223500 ext. 1277;

Fax. +524433273584 


\begin{abstract}
This paper presents an optimization strategy based on mathematical programming to solve resource management problems regarding hospitalization of sick patients, considering emergency scenarios, such as those that can occur in a pandemic. This optimization strategy is based on the use of efficient optimization tools in solving complex problems in which other strategies are not efficient. The mathematical modeling of epidemiological phenomena is a very useful tool to predict the direction of a disease, as well as to adequately and timely manage the available resources and thus save as many lives as possible. This work uses a mathematical model formulation based on deterministic optimization developed in general algebraic modelling system (GAMS) environment. The main user interface has been developed in a Microsoft ${ }^{\mathrm{TM}}(\mathrm{MS})$ Excel worksheet, which is familiar to many users. The linking code to send values from MS Excel to GAMS has been programmed in visual basic for applications (VBA) and it uses GAMS data exchange (GDX) files. The proposed optimization methodology is applied to case studies based on data obtained from affected people by severe acute respiratory syndrome coronavirus 2 (SARSCoV-2), which is the virus that causes coronavirus disease 2019 (COVID-19). The distances were taken as example from severely affected cities in USA. The obtained results offer attractive alternatives for the specified objective function in an acceptable computation time.
\end{abstract}

Keywords: Deterministic optimization strategy; Mathematical model formulation; GAMS; MS Excel; SARS-CoV-2; COVID-19. 


\section{INTRODUCTION}

At present, proper management of available natural resources (Pimentel et al. 1994) is essential to guarantee the sustainability of the lifestyle despite population growth (Ehrlich and Holdren 1971) and the impact it has on the environment (Cropper and Griffiths 1994). For this purpose, different optimization strategies have been developed that allow proposing different alternative solutions to the environmental problem, from the distribution of supply chains (Bowling et al. 2011), processes for obtaining biofuels (Hernández-Pérez et al. 2019), adequate management of resources in habitational complexes (Fuentes-Cortés et al. 2015), production of conventional electric energy through alternative technologies (Serrano-Arévalo et al. 2020), as well as the management of urban solid waste (Santibañez-Aguilar et al. 2013) and the analysis of the water-energy-food nexus (González-Bravo et al. 2018).

It is worth noticing that there are many optimization approaches from which it is possible to develop methodologies and propose models that offer us the best possible solution for a specific problem. Figure 1 shows a general classification of the different optimization approaches and global search methods (Coello-Coello et al. 2002; Devillers 1996). In general, the use of the optimization techniques mentioned above can be simplified as follows: in the first instance, deterministic search strategies are used to solve linear programming (LP) or mixed integer nonlinear programming (MINLP) formulations (Costa and Oliveira 2001) and when they have very complicated problems (highly non-convex) metaheuristic or stochastic programming is used (Ponce-Ortega and Hernández-Pérez 2019). Another advantage of using a metaheuristic optimization algorithm is that multiple configurations can be analyzed with different values of the search variables. This is particularly useful for evaluating the performance of certain objectives in a multi-scenario analysis.

Most of the reported works that are mentioned above to propose alternative solutions to the environmental problem, are part of optimization strategies through the use of mathematical programming (Horst and Tuy 1990) and deterministic solution strategies (Ponce-Ortega and Santibañez-Aguilar 2019). Computational software that allows implementing mathematical models for optimization are based on the use of mathematical tools to find the optimum of a specific problem. Two of the most commonly used commercial optimization programs are General Algebraic Modeling System (GAMS) software (Brooke et al. 1992) and Matrix Laboratory (MATLAB). 
However, there are also solution proposals through methodological strategies in which evolutionary and differential evolution (DE) algorithms have been used to solve those problems, in which due to their complexity, it is not possible to apply conventional search strategies.

Metaheuristic optimization strategies are often referred as stochastic optimization due to the uncertain nature inherent in evolutionary algorithms (Diwekar 2008). The above is due to the fact that in all these evolutionary search strategies a randomness factor is used with which new individuals or possible sets of solution values are generated, with this and with the use of mutation operators it is avoided to get caught in local solutions. In order to avoid confusion, in this text the metaheuristic term will refer to the use of different evolutionary optimization algorithms and the stochastic term the uncertainty associated with the use of randomness in the method.

When a methodological strategy that intends to use both optimization approaches is proposed, deterministic and metaheuristic, it is necessary to establish a link between the algorithms of each approach. There are different alternatives for linking optimization algorithms, even if they are on different platforms or programs. In the proposal presented here, a code to link Microsoft ${ }^{\mathrm{TM}}$ (MS) Excel and GAMS software was developed in visual basic for applications (VBA) using GAMS data exchange (GDX) files. The used linking subroutine allows the transfer of parameter and variable values from one algorithm to another.

The optimization framework of the methodological strategy proposed here was developed in order to solve a complex problem in the distribution of resources. The case study where it is applied is to solve the problem of resource management in an emergency scenario such as that which occurs during a pandemic. The case study presented to test the effectiveness of the method is a resource distribution problem elaborated with real data from the current pandemic.

Severe acute respiratory syndrome coronavirus 2 (SARS-CoV-2) is the virus that causes coronavirus disease 2019 (COVID-19). According to the World Health Organization (WHO, 2020), the disease has now infected more than 25.3 million people and killed about 848,000 victims worldwide. In the United States, one of the countries most affected by the pandemic, there have been registered more than 6 million confirmed positive cases for SARS-CoV-2 and the COVID19 has claimed the life of more than 183,000 people only in the USA according to data of September 1, 2020. Analyzing the previous data, it can be seen that about a quarter of the people affected by the disease reside in the United States of America. Likewise, the concentration of the greatest number of positive cases is found in states of that country, such as New York State, with 
more that 439,000 confirmed cases and with more than 32,500 deaths from the disease. Another state hardly affected by this new disease is New Jersey, where it has almost 194,000 positive cases and almost 16,000 deaths. Under the current trend following the COVID-19 pandemic, health care systems have already been saturated as projected by simulations (Ferguson et al. 2020).

Likewise, there are some states in this country that do not have such a high rate of infections and therefore the total number of people affected by the disease is less. Some of these states are neighbors (such as Connecticut, Pennsylvania and Delaware) to those most affected, so the analysis of efficient patient transfer strategies can offer alternative solutions to save as many people as possible.

The present methodological proposal consists in an optimization strategy based on mathematical programming to obtain the best management of resources in scenarios of a health crisis considering hospital restrictions. The mathematical modeling of a phenomenon such as the distribution of resources in crisis scenarios can be extremely complex. Therefore, the use of a methodology that allows the use of both deterministic optimization and metaheuristic tools is necessary.

Recently, the solution of an optimization problem has been reported that consists of the optimal management of an essential resource, water (Hernández-Pérez et al. 2020). In that work, a general methodology is used that allows to link deterministic optimization with metaheuristics. However, one characteristic of crisis scenarios is that they are under inherent uncertainty. Therefore, the development of a new multi-objective optimization methodology that allows the use of deterministic optimization and metaheuristics considering uncertainty is essential for optimal resource management in crisis scenarios such as what occurs during a pandemic.

In the present methodological proposal that is shown in this work, it is proposed to link the computer programs that allow the use of deterministic optimization in GAMS and metaheuristics through a DE algorithm programmed in MS Excel. The link between these programs is carried out through the action of a code or subroutine developed in the VBA environment. Likewise, it offers the user a simple and easily manageable interface developed in a program with which many users are accustomed.

\section{METHODOLOGY ALGORITHMS}

As mentioned previously, mathematical programming is a tool that consists in the representation of phenomena in models for their subsequent analysis and optimization. 
Optimization is a strategy to search for the best values that a decision variable (DV) can take with which the best possible performance of an objective function (OF) is achieved. However, the set of possibilities of configurations of a process (including those of transport) can become very exhaustive due to the large number of variables, equations, parameters and restrictions that are involved in the mathematical model. For this, the implementation and solution of these mathematical models in a computational tool is essential.

Optimization strategy programming is possible from a root language such as C++ and Java. However, there are currently a variety of commercial programs with deterministic optimization tools, such as Lindo and Gurobi. MS Excel can also be used to contain a metaheuristic optimization program (Hernandez-Perez et al. 2020) so it can be used by itself for linking with the simulation program and optimizing the problem using parameters specified by the user.

In this paper, a linking program code (LPC) was implemented to link the GAMS program with the MS Excel through VBA code. This strategy is done in order to send and receive data (like DV and OFs values) from the deterministic optimization algorithm (DOA) that is carried out in GAMS, with the metaheuristic optimization algorithm (MOA) that is previously programmed in MS Excel. Likewise, a stochastic optimization algorithm (SOA) is proposed from which uncertain parameters values (UPV) are created by the action of a random values generator (RVG) code, in this way the uncertainty is incorporated into the proposed optimization approach.

The methodological strategy proposed in this work consists of three fundamental parts, the MOA, the DOA and the SOA, each of which is described below:

- Deterministic optimization algorithm: The DOA is where the mathematical model is contained that is successively solved in GAMS software from the values that the MOA generates. The DOA reads the values of the DVs that the MOA generates through the LPC.

- Stochastic optimization algorithm: The SOA is the part of the methodological proposal, developed in VBA environment, that is responsible for incorporating the uncertainty from the UPV that creates the RVG code. The SOA creates a determined number of UPV calling RVG code for each set of DV values. Therefore, a large number of new functions must be analyzed in a statistical treatment and in this way select the values of the OFs that will be sent through the LPC to the MOA for its performance evaluation.

- Metaheuristic optimization algorithm: The MOA is the algorithm based on DE previously programmed in MS Excel. The MOA generates the sets of values (individuals) 
of the DV that may be the optimal solution. Likewise, it is in the MOA where the evaluation of the OFs is carried out in order to select the sets of values with the best performance and propose new values for the next iteration (generation).

These optimization algorithms are independent and are commonly used separately, however, due to the complexity of the case study that is addressed in this work, a hybrid methodology is developed that allows the use of deterministic optimization simultaneously with the metaheuristic optimization tools, while incorporating uncertainty by a stochastic algorithm. Each of the algorithms that are part of the methodology are described in detail below.

\section{GENERAL OPTIMIZATION APPROACH}

In this work, a stochastic hybrid (deterministic and metaheuristic) multi-objective optimization algorithm is developed. The general optimization approach is shown in Figure 2. The proposed methodology consists of the steps described below.

1. DV values generation by MOA: The MOA proposes sets of DV values that may be the optimal solution. The values proposed by the MOA are based on the user's specifications regarding the nature of the variable (continuous or integer) and their manipulation intervals. It is in the MOA where the number of sets of values of the DVs (population size, PS) and the number of iterations (maximum number of generations, MNG) are declared. Likewise, in the MOA it is possible to declare inequality restrictions and parameters for the operation of the metaheuristic algorithm.

2. UPV creation by RVG: For each set of DV values proposed by the MOA, the RVG assigns a specific number of UPV. The UPV number is specified by the user in the SOA programming. After the execution of the RVG code, the SOA assigns the UPVs to variables of the MOA that together with the DV values are sent to the DOA.

3. Sending DV values by the LPC: The values of the DVs proposed by the MOA and the UPV created by RVG are sent to the SOA by the action of the LPC. For this purpose, the LPC is composed of a set of subroutines or macros developed in VBA that allow the exchange of data between the MOA and the DOA, that is, between the GAMS software and the MS Excel platform. The set of macros that allow the linking of programs can be complicated, therefore, if a more detailed explanation of the particular methodology for linking the MOA with the DOA is required, more detailed information about it can be found in Hernández-Pérez et al. (2020). 
4. OF values calculation by DOA: In DOA, the single-objective optimization process is carried out. From the values of the DV proposed by the MOA and the UPV created by the RVG of the SOA, the deterministic optimization process of a single-objective is carried out considering these variables as parameters in each iteration of DOA in GAMS. The calculation of the OF in the DOA is made for each individual in the population and for each UPV.

5. Importation of the OF value by the LPC: After the calculation of the OF value in the DOA, this value is sent back to the SOA by the LPC.

6. OF statistical treatment in the SOA: Once the OF values for each set of DV and UPV are calculated and imported into the SOA. After that, a statistical treatment is carried out with the values of the OFs. This statistical treatment consists of calculating the mean of the set of values of the OFs. This new OF is called statistical objective function (SOF).

7. SOFs performance evaluation in MOA: Finally, for each individual in the population in each iteration, performance evaluation of the SOFs calculated by the SOA is made. For this purpose, the SOF is considered as one of the OFs in the MOA. In this part of the proposed methodology, the performance evaluation of the OFs is made in order to propose the values of the new individuals according to the selected metaheuristic optimization procedure.

By following the steps mentioned above, it is possible to use deterministic optimization simultaneously with metaheuristic tools and consider uncertainty. This methodology can be applied in solving the problem of optimal resource management for health crisis scenarios such as those that occur during a pandemic.

\section{CASE STUDY: OPTIMAL RESOURCE MANAGEMENT IN HEALTH CRISIS}

In order to test the effectiveness of the methodological proposal that is explained in this work, a case study has been selected: the solution to the problem of resource distribution in scenarios of uncertainty due to health crises such as those that occur in a pandemic.

For the specific case study addressed in this work, real data from the current pandemic caused by SARS-CoV-2 have been used. According with WHO, most people (about 80\%) recover from the disease without needing special treatment, and for the majority (especially for children and young adults) illness due to COVID-19 is generally minor. However, for some people it can cause serious illness. Around 1 in every 5 people who are infected with COVID-19 develop 
difficulty in breathing and require hospital care and about $4 \%$ end up in the intensive care units (ICUs). Most of those in intensive care require ventilators. Auld et al. (2020) found that $29.7 \%$ of COVID-19 patients who required ventilators died.

According with COVID-19 information from Centers for Disease Control and Prevention (CDC), current hospital capacity estimates are presented in Table 1. Estimates of hospital capacity were available at the national and state levels and includes data from April 1 to July 14, 2020. Data displayed on this table were submitted directly to CDC's National Healthcare Safety Network (NHSN) and do not include data submitted to other entities contracted by or within the federal government. These estimates used data that hospitals submitted to the NHSN COVID-19 Module and statistical methods that included weighting (to account for non-response) and multiple imputation (to account for missing data). The estimates (number and percentage) are shown along with $95 \%$ confidence intervals that reflect random error (cdc.gov/nhsn/covid19).

To know the current deficit or capacity of each state, it is necessary to calculate the number of COVID-19 patients who require specific medical care. The number of patients requiring a hospital bed or ICU is calculated from a balance to obtain active cases using data from confirmed cases, recovered cases and deaths. The active cases are calculated using expression shown in

\section{Equation 1.}

$$
\text { Active Cases }=\text { Confirmed }- \text { Recovered }- \text { Deaths }
$$

As mentioned above, the specific medical care for COVID-19 patients can be mainly of two types, hospital beds and ICUs. For the particular case study that is addressed in this work, these two necessary medical resources are analyzed, each of which is described below:

- Medical Resource 1: The first medical equipment necessary for those COVID-19 patients who require hospitalization is a hospital bed. To calculate the number of people who require a hospital bed, the number of active cases is multiplied by an estimation factor (E1). The value of $\mathrm{E} 1$ is 0.2 . The value of the estimation factor E1 is determined from the information provided by the WHO. The number of hospital beds needed is calculated using Equation 2.

$$
\text { Beds Needed }=E 1 *(\text { Active Cases })
$$

Using the information in Table 1 corresponding to the availability of hospital beds in each location and Equation 2, Table 2 is constructed. 
- Medical Resource 2: From COVID-19 patients requiring a hospital bed, a percentage require an ICU. To calculate the number of people who require an ICU, the number of active cases is multiplied by another estimation factor (E2). The value of E2 is 0.04 . The value of the estimation factor E2 is determined from the recently reported statistical analyzes (Auld et al., 2020). The number of ICUs needed is calculated using Equation 3.

$$
\text { ICUs Needed }=E 2 * \text { (Active Cases) }
$$

Using the information in Table 1 corresponding to the availability of ICUs in each location and Equation 3, it is constructed Table 3.

The balance of patients who requires specific medical care (medical resource 1 or medical resource 2) is made from the data of confirmed cases, recovered and deaths in each location. The data and active patient balance are the same for medical resource 1 (Table 2) and medical resource 2 (Table 3). From the balance of patients, the number of active cases is obtained. With the number of active cases and using the estimation factor for each medical resource, the need for each one is obtained. Subtracting this value from the availability of each medical resource, the capacity or deficit in each location is obtained.

Because E1 and E2 are estimation factors, they are uncertain. However, due to the random behavior inherent in a phenomenon such as a pandemic, there are many uncertain values. To consider all of them, the values that are considered uncertain in this case study are those corresponding to the number of patients who require hospitalization either for medical resource 1 or for medical resource 2 and who are on demand in hospitals originally. In this way, the deficit or capacity values obtained are used as a basis to generate uncertain values around them.

On the other hand, an important aspect to analyze in the case study presented here is the amount of new equipment to be acquired, both for hospital beds and ICUs in the destination hospitals that will receive patients from localities in over-demand. The number of new equipment purchased for medical resource 1 and for medical resource 2 is variable and the decision of the amount determines the best solution. The analysis of this aspect together with the information obtained from the transport of patients, composes by itself the model of a problem to be optimized.

The details of the creation of the uncertain parameters are described in detail in SOA, and how they are used to calculate the optimal distribution of transported patients are described in detail in DOA, while the variable capacity of destination hospitals is explained in MOA description. All of them in the following parts of this text. 
Due to the complexity involved in both, the modeling and the optimization process, a new hybrid strategy is necessary. In the described method, the intervention of deterministic optimization and metaheuristic tools is required to propose an alternative solution. Likewise, it is necessary to introduce randomness in the method, due to the uncertain nature with which diseases behave.

In the optimization model that is proposed in this case study, two OFs are considered, one social and the other economic. The first OF corresponds to the primary interest in a health crisis scenario, save lives. The second OF corresponds to the economic available resource to attend the pandemic.

The social OF is directly related to the number of patients who require special medical care and is adequately treated according to their situation, in other words, the possibility of increasing the number of lives saved is by guaranteeing adequate medical attention to the greatest number of people who require it. To achieve this, there are two options: transfer patients who require intensive care to a hospital that has the capacity to attend them, or acquire new equipment at the hospital that is on demand. However, the number of patients in an over-demand hospital (origin hospital) is uncertain and the capacity to care for them is variable depending on the equipment acquired by each host hospital (destination hospital). The probability of saving lives increases by minimizing the number of neglected patients as is defined on OF1 specification in MOA.

In the formulation of the model that corresponds to the MOA, it is considered that the hospitals in the over-demand states do not have enough space to install the medical equipment that they could obtain, so the purchase of new equipment is restricted to the host hospitals. The above converts the number of equipment obtained in the host hospitals in the DVs of the MOA model and the excess demand of saturated hospitals in the UPV of the SOA model.

\section{DOA MODEL FORMULATION}

The DOA is the step of the methodology that allows both to raise a part of the mathematical model and to carry out the deterministic optimization process. One of the parts of the DOA is the representation of a phenomenon that occurs in reality through a mathematical model. This mathematical model consists of a set of parameters, variables and equations that are solved simultaneously in the deterministic optimization process. The other part of DOA consists of the optimization process that is carried out by the GAMS software. This optimization process is carried out considering the model as a single-objective problem. Therefore, if the original approach 
consists of a multi-objective optimization problem, it is necessary to add the calculation and the value of the others OFs to the MOA.

For the particular case study presented in this work, it is necessary to address the transport model. There are some examples that can be used as a base, such as the McCarl Transport Model. This and other examples can be found in the GAMS user guide section (gams.com).

In general, the transport model consists of finding the optimal distribution of resources according to some known values of the problem. The information that is entered into the model that works as a known parameter consists of: the distance between the locations, a demand (hospital capacity in this case) and an offer (patients requiring hospitalization). Likewise, in the transportation model a fixed transportation cost is used. For the particular case study addressed in this work, the study of two states with over-demand of patients (New York and New Jersey) and three states with the capacity to serve said patients (Connecticut, Pennsylvania and Delaware) is carried out. The superstructure of the possible distribution of patients among the states selected in the study is shown in Figure 3. Table 4 shows the used distance between states, expressed in miles. For the particular case study that is presented in this work, the DOA specifications are as shown below:

- DOA sets: origin hospitals (i), destination hospitals (j).

- DOA parameters: patients who exceeded the maximum capacity of the origin hospital i $\left(a_{i}\right)$, patients that can be treated at the destination hospital $\mathrm{j}$ due to the new equipment acquired $\left(b_{i}\right)$, distances between origin hospital $i$ and destination hospital $j\left(d_{i j}\right.$ in $\$ /$ mile//case), transport cost ( $c_{1}$ in $\$ /$ case/1,000 miles). The value of $\mathrm{c} 1$ is 30 US\$ per mile for medical resource 1 and 50 US\$ per mile for medical resource 2.

- DOA variables: number of patients who are transferred from hospital origin i to hospital destination $\mathrm{j}\left(\mathrm{x}_{\mathrm{ij}}\right)$.

- DOA OF: The OF of the DOA is expressed in Equation 4:

$$
\operatorname{Min} f\left(x_{i j}\right)=c_{1} \sum_{i=1} \sum_{j=1} d_{i j} x_{i j}
$$

- DOA restrictions: The restrictions of the OF of the DOA depend of the medical equipment. For medical resource 1 , the restrictions are defined as $C_{1}$ and $C_{2}$, for medical resource 2 the restrictions are defined as $\mathrm{C}_{3}$ and $\mathrm{C}_{4}$. 


$$
\begin{aligned}
C_{1} & \equiv \sum_{j=1}^{m} x_{i j} \leq a_{i} \forall i \\
C_{2} & \equiv \sum_{i=1}^{n} x_{i j} \geq b_{j} \forall j \\
C_{3} & \equiv \sum_{j=1}^{m} x_{i j} \geq a_{i} \forall i \\
C_{4} & \equiv \sum_{i=1}^{n} x_{i j} \leq b_{j} \forall j \\
C_{5} & \equiv x_{i j} \geq 0 \forall i, j
\end{aligned}
$$

Following the mathematical model presented above, it is possible to solve the patient transport problem using deterministic optimization through GAMS software. For the DOA, in this case study, a LP model using the CPLEX solver was specified.

\section{SOA PARTS AND SEQUENCE}

The SOA is the part of the methodology that allows considering the uncertainty through the creation of UPV by the RVG code. Likewise, in the SOA, the DOA call and the import of OF value are carried out in each deterministic optimization process in GAMS. With the OFs values of multiple sets of DV values and UPV, a statistical treatment is made to calculate the arithmetic mean that composes the SOF. Subsequently, the SOF is declared as OF in the MOA for the evaluation of its performance and with these new values are proposed for the DVs in the next iteration. Therefore, MOA calls the SOA for each individual, which in turn calls the DOA for each UPV created by the RVG code. The SOA consists of the following key parts:

1. Declaration of DV and OF: First, it is necessary to declare the DVs that are part of the SOA, these search variables correspond to the DV values that are generated in the MOA. The OF that is declared in the SOA is the one that will be obtained in each DOA optimization process.

2. RVG code: The RVG code creates a set number of UPVs to introduce the uncertainty in the method. These UPVs take random values in a previously established interval. For each of these UPVs, the DOA is called through the LPC.

3. SOF calculation: Once the value of multiple OFs in the DOA has been calculated, they are imported into the SOA through the LPC. After that, a statistical treatment is carried out 
that consists of calculating the arithmetic mean of said OFs. This arithmetic mean that is calculated conform the SOF and is declared as OF in the MOA.

Each of the parts of the SOA followed in the sequence mentioned above, allows the incorporation of uncertainty into the methodology. This through the creation of UPV by the RVG and the subsequent statistical analysis of the OFs values obtained in each DOA optimization process to calculate the SOF, which is declared as OF in the MOA.

For the particular case study that is presented in this work, the SOA specifications are as shown below:

To introduce uncertainty into the problem, the number of patients requiring hospitalization are considered as the uncertain parameters. The manipulation range of the RVG code for the UPV is $5 \%$ around the original value of the uncertain parameter.

For the particular case study analyzed in this work, UPVs are created by the RVG code around the base value of the number of patients who require special medical care (either hospital beds or ICUs). Depending on the medical resource to be analyzed, the data in the column "Deficit/Capacity" are taken.

In case of the analysis of medical resource 1 (hospital beds), the data from Table 2 are taken. Following the indications above for the UPV, the RVG code will propose integer random values for the numerical constant $(18,374)$ between 17,455 and 19,293 (18,374 $\pm 5 \%)$ for the case of New York and integer random values for the numerical constant (10,075) between 9,571 and and $10,579(10,075 \pm 5 \%)$ for the case of New Jersey.

In case of the analysis of medical resource 2 (ICUs), the data from Table $\mathbf{3}$ are taken. Following the indications above for the UPV, the RVG code will propose integer random values for the numerical constant $(8,329)$ between 7,913 and 8,745 $(8,329 \pm 5 \%)$ for the case of New York and integer random values for the numerical constant $(3,476)$ between 3,302 and 3,650 $(3,476 \pm 5 \%)$ for the case of New Jersey.

From the uncertain values and the capacity of each destination hospital, it is possible to determine the number of patients that will be rejected. Minimizing this function is expected to increase the chance of saving lives. The mathematical relationship to determine the number of rejected patients is expressed in Equation 10 for medical resource 1. 


$$
\operatorname{Min} g\left(a_{i}, b_{j}\right)=\sum_{j=1} b_{j}-\sum_{i=1} \mu a_{i}
$$

Subject to:

$$
C_{4} \equiv \sum_{j=1} b_{j} \geq \sum_{i=1} \mu a_{i}
$$

The mathematical relationship to determine the number of rejected patients is expressed in Equation 12 for medical resource 2.

$$
\operatorname{Min} g\left(a_{i}, b_{j}\right)=\sum_{i=1} \mu a_{i}-\sum_{j=1} b_{j}
$$

For medical resource 2, subject to:

$$
C_{4} \equiv \sum_{i=1} \mu a_{i} \geq \sum_{j=1} b_{j}
$$

Where:

$\mathrm{g}=$ neglected patients

$\mathrm{i}=$ origin hospitals

$\mathrm{j}=$ destination hospitals

$a_{i}=$ patients who exceeded the maximum capacity of the origin hospital $i$

$\mathrm{b}_{\mathrm{j}}=$ patients that can be treated at the destination hospital $\mathrm{j}$ due to the new equipment acquired

$\mu=$ uncertain parameter value (excess demand of saturated hospitals)

The SOF consists in the arithmetic mean, that is, the average of a set of numerical values, calculated by adding them together and dividing by the number of terms in the set. In this methodology, these numerical values correspond to the performance of the OF calculated in the DOA for each UPV created by the RVG code. Therefore, the number of OF values that will have this statistical treatment to calculate the SOF is equal to the number of UPVs (K).

$\mathrm{SOF}_{1}$ mathematical definition is expressed in Equation 14.

$$
S_{0} F_{1}(g)=\frac{1}{K} \sum_{k=1}^{K} g_{k}
$$

Where: 
$\mathrm{SOF}_{1}=$ statistical objective function 1 , the arithmetic mean of a set of $\mathrm{g}$.

$\mathrm{g}=$ neglected patients.

$\mathrm{k}=\mathrm{UPV}$ number $\mathrm{k}$.

$\mathrm{K}=$ set of numerical values (number of UPV created by RVG in SOA).

$\mathrm{SOF}_{2}$ mathematical definition is expressed in Equation 15.

$$
S O F_{2}(f)=\frac{1}{K} \sum_{k=1}^{K} f_{k}
$$

Where:

$\mathrm{SOF}_{2}=$ statistical objective function 2 , the arithmetic mean of a set of $\mathrm{f}$.

$\mathrm{f}_{\mathrm{k}}=$ uncertain OF calculated in the DOA using the UPV number $\mathrm{k}$, uncertain transport cost.

$\mathrm{K}=$ set of numerical values (number of UPV created by RVG in SOA).

Using SOF it is possible to analyze the average of a set of multiple OFs calculated by DOA for each UPV created in SOA. The SOF is only a part of the economical OF that must be declared in MOA.

\section{MOA DESCRIPTION}

The MOA is the tool that allows the solution of complex mathematical models with nonlinear equations and highly non-convex problems. One of the characteristics of metaheuristic optimization algorithms is that they allow the "what if" analysis to be carried out. The "what if" analysis is one of the most useful functions that the algorithms based on DE have, since they allow comparing the performance of the OFs with different values of the DVs and in different scenarios. In this work, the MOA is used to perform a "what if" analysis of different scenarios by changing DV values.

Part of the methodological strategy presented in this work is developed in MS Excel through VBA. MS Excel is a computer a software that allows to perform simple numerical (like accounting and financial) tasks due to its included functions, developed specifically to help create and work with spreadsheets. One of the strengths of MS Excel is that it gives its users the ability to customize their spreadsheets by programming their own functions, performing specific tasks, adjusted to the needs of each one, and that have not been included in the original package.

For developers who are looking for very specific objectives, or for a complexity greater than the calculation of common functions, the solution lies in the use of Visual Basic. It is a 
language with a relatively high degree of abstraction (which is considerably different from the machine language, the way in which the processor works) and which, like the one used in MS Excel, works by events (this means that the user must perform some action to trigger the execution of the program). VBA is the language of the MS operating system (Windows) that is used for program applications. Many of the programs and add-ons that are used in Windows are developed in this language so there are common elements that can be manipulated through this platform.

The combination of MS Excel and Visual Basic also has its limitations, and in no way can exceed the level of customization and precision possible through the creation of a program from scratch; but it is very effective and comfortable to the user. Due to the characteristics mentioned above, MS Excel represents a viable alternative for the development of stochastic optimization algorithms (Martin-Martin 2019).

For the particular case study that is presented in this work, the MOA specifications are as shown below:

- I-MODE algorithm: There are a wide variety of MOAs that can be used in the proposed optimization methodology. The selected MOA is the I-MODE algorithm (Sharma and Rangaiah 2013) developed in MS Excel through VBA. The multi-objective optimization metaheuristic method called I-MODE works with different termination criteria: chisquared termination criterion (ChiTC), steady state termination criterion (SSTC) and after MNG.

- MOA variables: The I-MODE variables correspond to the DVs that are mentioned in the case study explanation. For MOA purposes, only the number of patients that are capable of receiving the assigned hospitals or destination hospitals are considered as search variables. $\left(b_{1}, b_{2}\right.$ and $\left.b_{3}\right)$. The value of these variables will determine the OF performance. Regarding the search interval, it depends on the medical resource analyzed. In the case of medical resource 1 (hospital beds), a maximum increase of $10 \%$ of the current capacity of each locality is considered. Regarding medical resource 2 (ICUs), a maximum increase of $5 \%$ of the current capacity of each locality is considered.

- MOA algorithm parameters: For the optimization process, in this study the values for the parameters associated to the used I-MODE algorithm are the following: PS: 10 individuals, MNG: 100 generations, taboo list size (TLS): 50 individuals, taboo radius (TR): 0.01, crossover fraction $(\mathrm{CF})$ : 0.5, mutation fraction $(\mathrm{F})$ : 0.5 . 
- OF specification in MOA: In the MOA, the model is considered as a multi-objective optimization problem. The OFs that are considered correspond, one to a social aspect and the other to economic interests. The social OF consists of minimizing the number of patients who are left without medical attention, thereby seeking to increase the chances that a greater number of patients will have the necessary medical care. $\mathrm{OF}_{1}$ is expressed in Equation 16.

$$
\operatorname{Min} O F_{1}(g)=S O F_{1}(g)
$$

Where:

$\mathrm{OF}_{1}=$ objective function 1 declared in MOA, rejected patients.

$\mathrm{g}_{\mathrm{k}}=$ uncertain OF calculated in the MOA using the UPV number $\mathrm{k}$, uncertain rejected patients.

$b_{j}=$ patients that can be treated at the destination hospital $\mathrm{j}$ due to the new equipment acquired.

$\mathrm{k}=$ uncertain parameter (excess demand of saturated hospitals).

On the other hand, the economic OF consists of minimizing the total costs, which are composed of the costs of transporting patients and the costs allocated to the acquisition of specialized medical equipment. $\mathrm{OF}_{2}$ is expressed in Equation 17.

$$
\operatorname{Min} O F_{2}(b, f)=c_{2} \sum_{j=1} b_{j}+S O F_{2}(f)
$$

Where:

$\mathrm{OF}_{2}=$ objective function 2 declared in MOA, total cost.

$\mathrm{SOF}_{2}=$ statistical objective function 2 , the arithmetic mean of a set of $\mathrm{f}$.

$\mathrm{j}=$ destination hospitals.

$b_{j}=$ patients that can be treated at the destination hospital $j$ due to the new equipment acquired.

$c_{2}=$ cost of the new equipment acquired (US\$/unit). The value of $c_{2}$ is 1,200 US $\$$ for medical resource 1 and 27,000 US\$ for medical resource 2.

The two OFs that are specified in the MOA must correspond to those that are expressed in Equation 9 and in Equation 10. Likewise, the goal for each OF in the MOA for this particular study case must be minimizing. 
In general, the fundamental parts of the MOA have been shown, these specifications can be modified according to the characteristics of the problem in the case study to be solved as well as the specifications necessary in the evolutionary algorithm selected as MOA.

\section{LPC MODULES}

To successfully link GAMS to MS Excel, the use of a GDX file is necessary using code written in VBA. A linking code usually includes different instructions that are grouped into modules. These modules are sub routines that perform a specific task. Subsequently, a main module calls each sub routine in the proper order for them to work correctly. These lines of code are grouped into sub routines that are called by a main module. More detailed information about it can be found in Hernández-Pérez et al. (2020).

\section{RESULTS AND DISCUSSION}

This section presents the results of the stochastic hybrid (deterministic and metaheuristic) multi-objective optimization approach to solve the resource management problem in health crisis scenarios under uncertainty such as those that occur during a pandemic. The methodological strategy presented in this work is made up of three optimization algorithms, the DOA, the SOA and the MOA. The aforementioned algorithms communicate and send and receive different values of variables and parameters. Likewise, they are run in a simultaneous and orderly manner to solve a complex problem such as the one presented in the specific case study.

The entire optimization framework was performed on an Intel ${ }^{\mathrm{TM}}$ Core $^{\mathrm{TM}}$ i7-6700HM CPU @ $2.6 \mathrm{GHz}, 32 \mathrm{~GB}$ computer. The computation time to solve the specific case study considering the analysis of medical resource 1 (hospital beds) was $37.07 \mathrm{~min}$. On the other hand, the computation time to solve the specific case study considering the analysis of medical resource 2 (ICUs) was $40.84 \mathrm{~min}$.

The termination criteria specified in the selected MOA (I-MODE) was MNG. Once the MOA reaches the MNG, it offers results with which it is possible to build the graphs presented in Figure 4 for the analysis of medical resource 1 (hospital beds) and in Figure 5 for analysis of medical resource 2 (ICUs). The series of points shown in Figure $\mathbf{4}$ and Figure 5 all correspond to optimal solutions. Only feasible solutions from the best/first Pareto-optimal front in the last generation are presented. From this set of points, the decision maker can select the one that best reconciles both OFs, the social and the economic. The points that best reconcile the performance 
of the OFs are usually on the curve that is formed in the graph. These points have been marked as $\mathrm{A}$ in the Figure 4 and as B in the Figure 5.

The optimal values of the DVs and the performance of the OFs obtained with these values are presented in Table 5. The values presented in said table are of points A and B and correspond to the analysis of medical resource 1 and medical resource 2 , respectively.

Likewise, with the values of the DVs that are presented in Table 5 and with the current demand without considering the uncertainty in the demand for medical equipment, Table 6 is obtained for the analysis of medical resource 1 and Table 7 for the analysis of the medical resource 2. These tables show the optimal distribution of patients according to the excess demand in the origin hospitals and the capacity to attend to the same patients in the destination hospitals.

As can be seen in Table 6 and in Table 7, the optimal distribution of patients corresponds to the one that allocates the largest number of patients from the places with the highest demand to those who have the capacity to receive them and who are within a short distance. However, the balance used can be updated and analyzed different scenarios as the pandemic progresses.

\section{CONCLUSIONS}

This paper has presented a new multi-objective stochastic optimization framework based on deterministic optimization and metaheuristic tools to solve complex problems considering uncertainty. Also, the user is offered a familiar and easy-to-use interface to quickly manipulate the values and make appropriate decisions in each possible scenario that the advance of the pandemic may form.

The methodology presented in this work consists of solving the problem using different algorithms: DOA, SOA and MOA. The MOA proposes values of the DVs, the total capacity to attend patients with the current capacity plus the capacity due to the medical equipment acquired. The SOA creates random values of the uncertain parameters to bring the uncertainty to the model. Based on the values of the DV of the MOA and the UPV of the SOA, the DOA solves the patient distribution from the origin hospitals on demand to the destination hospitals that can serve them, either with the medical equipment available or acquiring new.

To test the effectiveness of this method, the case study of the optimal distribution of resources in health crisis scenarios such as those that occur during a pandemic is addressed. The data used to make the analysis of the specific case study correspond to those of the current COVID19 pandemic caused by SARS-CoV-2. It also considers a balance of patients who require 
hospitalization to update the distribution requirements. If this methodology is used, it is possible to stablish the best patient distribution.

For the solution of the scenarios that are addressed in the case study, real data reported by the WHO of the COVID-19 pandemic were used. These data were collected on September 1, 2020; however, it is possible to update the numbers and run the optimization methodology presented here again. Likewise, it is possible to change the data of distances between locations, capacities and demands. As for the uncertainty, a different interval can be manipulated to create uncertain parameters.

Optimization functions obey the fulfillment of two simultaneous objectives, one social and the other economic. The social objective corresponds to minimizing the number of rejected patients, thereby increasing the chances of saving lives. While the economic objective corresponds to minimizing the total costs of both transportation and acquisition of medical equipment (hospital beds and ICUs). The results show the optimal configuration of the medical equipment acquired by analyzing medical resource 1 (hospital beds) and medical resource 2 (ICUs) as well as the adequate distribution of patients from hospitals on demand to destination hospitals that can attend them considering the uncertainty associated with changes in demand.

\section{NOMENCLATURE}

\section{Sets}

$\mathrm{i}=$ origin hospitals

$\mathrm{j}=$ destination hospitals

$\mathrm{k}=$ uncertain parameter number

$\mathrm{K}=$ set of numerical values (number of UPV created by RVG in SOA)

\section{Variables and Parameters}

$\mathrm{a}_{\mathrm{i}}=$ patients who exceeded the maximum capacity of the origin hospital $\mathrm{i}$ (using $\mu$, UPV in SOA, parameter in DOA)

$\mathrm{b}_{\mathrm{j}}=$ patients that can be treated at the destination hospital $\mathrm{j}$ due to the new equipment acquired (variable in MOA, parameter in DOA)

$\mathrm{c}_{1}=$ transport cost $(\$ /$ case $/ 1,000$ miles) (parameter in DOA)

$\mathrm{c}_{2}=$ cost of the new equipment acquired (\$/unit) (parameter in MOA)

$\mathrm{d}_{\mathrm{ij}}=$ distances between origin hospital $\mathrm{i}$ and destination hospital $\mathrm{j}$ (\$/mile//case) (parameter in DOA) 
$\mathrm{X}_{\mathrm{ij}}=$ number of patients who are transferred from hospital origin $\mathrm{i}$ to hospital destination $\mathrm{j}$ (variable in DOA)

$\mu=$ uncertain parameter value (UPV in SOA), (excess demand of saturated hospitals)

\section{Functions}

$\mathrm{f}_{\mathrm{k}}=$ uncertain OF calculated in the DOA using the UPV number $\mathrm{k}$

$\mathrm{g}_{\mathrm{k}}=$ uncertain OF calculated in the SOA using the UPV number $\mathrm{k}$

$\mathrm{OF}_{1}=$ objective function 1 declared in $\mathrm{MOA}$, rejected patients

$\mathrm{OF}_{2}=$ objective function 2 declared in MOA, total cost

$\mathrm{SOF}_{1}=$ statistical objective function 1 , the arithmetic mean of a set of $\mathrm{g}$

$\mathrm{SOF}_{2}=$ statistical objective function 2 , the arithmetic mean of a set of $\mathrm{f}$

\section{Estimation Factors}

E1 = Estimation factor to calculate beds needed (0.2) (parameter in patients balance)

E2 = Estimation factor to calculate ICUs needed (0.04) (parameter in patients balance)

\section{ACRONYMS}

$\mathrm{CDC}=$ Center for Disease Control and Prevention

$\mathrm{CF}=$ Crossover Fraction

ChiTC $=$ Chi-squared Termination Criteria

COVID-19 = Coronavirus Disease 2019

DOA $=$ Deterministic Optimization Algorithm

$\mathrm{DV}=$ Decision Variables

GAMS $=$ General Algebraic Modeling System

$\mathrm{F}=$ Mutation Fraction

GDX $=$ GAMS Data eXchange

$\mathrm{ICU}=$ Intensive Care Unit

LP $=$ Lineal Programing

LPC $=$ Linking Program Code

MINLP = Mixed-Integer Non-Linear Programming

MNG = Maximum Number of Generations

MOA = Metaheuristic Optimization Algorithm

$\mathrm{MS}=$ Microsoft $^{\mathrm{TM}}$

NHSN $=$ National Healthcare Safety Network 
$\mathrm{OF}=$ Objective Function

PS = Population Size

$\mathrm{RVG}=$ Random Values Generator

SARS-CoV-2 $=$ Severe Acute Respiratory Syndrome Coronavirus 2

SOA $=$ Stochastic Optimization Algorithm

SOF $=$ Statistical Objective Function

SSTC $=$ Steady State Termination Criteria

TLS $=$ Taboo List Size

$\mathrm{TR}=$ Taboo Radius

UPV $=$ Uncertain Parameters Values

VBA = Visual Basic for Applications

WHO $=$ World Health Organization

\section{Author Information}

\section{Corresponding Author}

*Ponce-Ortega Jose Maria Tel. +52-443-3223500. Ext. 1277. Fax. +52-443-3273584. E-mail: jmponce@umich.mx

Notes

The authors declare no competing financial interest.

\section{Acknowledgements}

This project was funded by CIC-UMSNH.

The authors also acknowledge to Professors G.P. Rangaiah and S. Sharma for facilitating the I-MODE code.

\section{REFERENCES}

Auld S, Caridi-Scheible M, Blum JM, Robichaux CJ, Kraft CS, Jacob JT, Jabaley CS, Carpenter D, Kaplow R, Hernandez AC, Adelman MW (2020) ICU and ventilator mortality among critically ill adults with COVID-19. Emory COVID-19 Quality and Clinical Research Collaborative. https//doi.org/10.1101/2020.04.23.20076737

Bowling IA, Ponce-Ortega JM, El-Halwagi MM (2011) Facility location and supply chain optimization for a biorefinery. Ind. Eng. Chem. Res. 50 (10), 6276-6286. https//doi.org/10.1021/ie101921y 
Brooke A, Kendrick D, Meeraus A (1992) Release 2.25 GAMS (General Algebraic Modeling System) A User's Guide. Boyd \& Fraser Publishing Co. Danvers, Massachusetts.

Coello-Coello CA, Van-Veldhuizen DA, Lamont GB (2002) Evolutionary algorithms for solving multi-objective problems. Kluwer Academic. New York, NY, USA.

Costa L, Oliveira P (2001) Evolutionary algorithms approach to the solution of mixed integer nonlinear programming problems. Comput. Chem. Eng. 25 (2-3), 257-266. https//doi.org/10.1016/S0098-1354(00)00653-0

Cropper M, Griffiths C (1994) The Interaction of Population Growth and Environmental Quality. Am. Econ. Rev. 84(2), 250-254. https//doi.org/10.2307/2117838

Devillers J (1996) Genetic algorithms in molecular modeling. Academic Press. San Diego, CA.

Diwekar U (2008) Introduction to applied optimization (Vol. 22). Springer Science \& Business Media. Clarendon Hills, IL, USA.

Ehrlich P, Holdren, J (1971) Impact of population growth. Sci. 171(3977), 1212-1217. https//doi.org/10.2307/1731166

Ferguson NM, Laydon D, Nedjati-Gilani G, Imai N, Ainslie K, Baguelin M, Bhatia S, Boonyasiri A, Cucunubá Z, Cuomo-Dannenburg G, Dighe A (2020) Impact of non-pharmaceutical interventions (NPIs) to reduce COVID-19 mortality and healthcare demand. Avaliable at: www.if.ufrgs.br/if/wp-content/uploads/Imperial-College-COVID19-NPI-modelling-1603-2020.pdf

Fuentes-Cortés LF, Ponce-Ortega JM, Nápoles-Rivera F, Serna-González M, El-Halwagi MM (2015) Optimal design of integrated CHP systems for housing complexes. Energy Convers. Manag. 99, 252-263. https//doi.org/10.1016/j.enconman.2015.04.036

González-Bravo R, Mahlknecht J, Ponce-Ortega JM (2018) Water, food and power grid optimization at macroscopic level involving multi-stakeholder approach. Energy Procedia. 153, 347-352. https//doi.org/10.1016/j.egypro.2018.10.013

Hernandez-Perez LG, Alsuhaibani AS, Radwan N, El-Halwagi MM, Ponce-Ortega JM (2020) Structural and operating optimization of the methanol process using a metaheuristic technique. ACS Sustainable Chem. Eng. 8 (8), 3135-3150. https//doi.org/10.1021/acssuschemeng.9b05981

Hernández-Pérez LG, Lira-Barragán LF, Ponce-Ortega JM (2020) Hybrid multiobjective optimization using deterministic and metaheuristic techniques for flowback water reusing 
in hydraulic fracturing processes. Ind. Eng. Chem. Res. 59, 34, 15298-15308 https://doi.org/10.1021/acs.iecr.0c02867

Hernández-Pérez LG, Sánchez-Tuirán E, Ojeda KA, El-Halwagi MM, Ponce-Ortega JM (2019). Optimization of microalgae-to-biodiesel production process using a metaheuristic

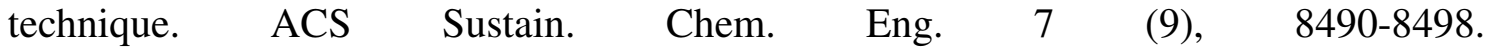
https//doi.org/10.1021/acssuschemeng.9b00274

Horst R, Tuy H (1990) Global Optimization: Deterministic Approaches. Springer-Verlag. 1990. Berlin Heidelberg. https//doi.org/10.1007/978-3-662-02598-7

Martin-Martin M (2019) Introduction to software for process simulation. CRC Press. USA. ISBN: 978-1138324220

Pimentel D, Harman R, Pacenza M, Pecarsky J, Pimentel M (1994) Natural resources and an optimum human population. Population Environ. 15 (5), 347-369. https//doi.org/ 10.1007/BF02208317

Ponce-Ortega JM, Hernández-Pérez LG (2019) Optimization of process flowsheets through metaheuristic techniques. Springer. Switzerland. ISBN 978-3-319-91722-1

Ponce-Ortega JM, Santibañez-Aguilar JE (2019) Strategic planning for the sustainable production of biofuels. Elsevier. Oxford, United Kingdom. ISBN10: 0128181788

Santibañez-Aguilar JE, Ponce-Ortega JM, Gonzáles-Campos JB, Serna-Gonzáles M, El-Halwagi MM (2013) Optimal planning for the sustainable utilization of municipal solid waste. J. Waste Manag. 33 (12), 2607-2622. https//doi.org/10.1016/j.wasman.2013.08.010

Serrano-Arévalo TI, Juárez-García M, Ponce-Ortega JM (2020) Optimal planning for satisfying future electricity demands involving simultaneously economic, emissions, and water concerns. Process Integr. Optim. Sustain. https//dor.org/10.1007/s41660-020-00125-8

Sharma S, Rangaiah GP (2013) An improved multi-objective differential evolution with a termination criterion for optimizing chemical processes. Comput. Chem. Eng. 56, 155173. https//doi.org/10.1016/j.compchemeng.2013.05.004 
TABLES

Table 1. Hospital capacity estimates.

\begin{tabular}{|c|c|c|c|c|c|c|}
\hline $\begin{array}{c}\text { HOSPITAL } \\
\text { CAPACITY }\end{array}$ & \multicolumn{3}{|c|}{ Beds } & \multicolumn{3}{c|}{ ICUs } \\
\hline Location & Available & Occupied & $\begin{array}{c}\% \\
\text { Occupied }\end{array}$ & Available & Occupied & $\begin{array}{c}\% \\
\text { Occupied }\end{array}$ \\
\hline New York & 47,995 & 31,533 & 65.70 & 8,263 & 4,396 & 53.20 \\
\hline New Jersey & 18,702 & 13,933 & 74.50 & 3,718 & 1,610 & 43.30 \\
\hline Connecticut & 9,093 & 5,983 & 65.80 & 1,618 & 772 & 47.70 \\
\hline Pennsylvania & 40,692 & 26,926 & 66.17 & 5,034 & 2,819 & 56.00 \\
\hline Delaware & 2,582 & 1,771 & 68.60 & 308 & 195 & 63.40 \\
\hline
\end{tabular}

Table 2. Patients balance for beds needed.

\begin{tabular}{|c|c|c|c|c|c|c|c|}
\hline $\begin{array}{l}\text { PATIENTS } \\
\text { BALANCE }\end{array}$ & \multicolumn{4}{|c|}{ Data } & \multicolumn{3}{|c|}{ Projection } \\
\hline Location & $\begin{array}{l}\text { Confirme } \\
\text { d }\end{array}$ & $\begin{array}{l}\text { Recovere } \\
\text { d }\end{array}$ & Deaths & $\begin{array}{l}\text { Active } \\
\text { Cases }\end{array}$ & $\begin{array}{l}\text { Beds } \\
\text { Needed }\end{array}$ & $\begin{array}{l}\text { Availabl } \\
\text { e }\end{array}$ & $\begin{array}{l}\text { Deficit/Capacit } \\
\text { y }\end{array}$ \\
\hline New York & 439,480 & 75,100 & 32,531 & 331,849 & 66,370 & 47,995 & $-18,374$ \\
\hline New Jersey & 193,740 & 33,912 & 15,945 & 143,883 & 28,777 & 18,702 & $-10,075$ \\
\hline Connecticut & 52,879 & 8,967 & 4,465 & 39,447 & 7,889 & 9,093 & 1,203 \\
\hline Pennsylvania & 139,376 & 109,900 & 7,764 & 21,712 & 4,342 & 40,692 & 36,350 \\
\hline Delaware & 17,429 & 9,381 & 605 & 7443 & 1,489 & 2,582 & 1,093 \\
\hline
\end{tabular}

Table 3. Patients balance for ICUs needed.

\begin{tabular}{|c|c|c|c|c|c|c|c|}
\hline $\begin{array}{l}\text { PATIENTS } \\
\text { BALANCE }\end{array}$ & \multicolumn{4}{|c|}{ Data } & \multicolumn{3}{|c|}{ Projection } \\
\hline Location & $\begin{array}{l}\text { Confirme } \\
\text { d }\end{array}$ & $\begin{array}{l}\text { Recovere } \\
\text { d }\end{array}$ & Deaths & $\begin{array}{l}\text { Active } \\
\text { Cases }\end{array}$ & $\begin{array}{l}\text { ICUs } \\
\text { Needed }\end{array}$ & $\begin{array}{l}\text { Availabl } \\
\text { e }\end{array}$ & $\begin{array}{l}\text { Deficit/Capacit } \\
\text { y }\end{array}$ \\
\hline New York & 439,480 & 75,100 & 32,531 & 331,849 & 13,274 & 8,263 & $-5,011$ \\
\hline New Jersey & 193,740 & 33,912 & 15,945 & 143,883 & 5,755 & 3,718 & $-2,037$ \\
\hline Connecticut & 52,879 & 8,967 & 4,465 & 39,447 & 1,578 & 1,618 & 41 \\
\hline Pennsylvania & 139,376 & 109,900 & 7,764 & 21,712 & 868 & 5,034 & 4,165 \\
\hline Delaware & 17,429 & 9,381 & 605 & 7,443 & 298 & 308 & 10 \\
\hline
\end{tabular}


Table 4. Distance between states in miles

\begin{tabular}{|l|l|l|l|}
\hline Distance & Connecticut & Pennsylvania & Delaware \\
\hline New York & 97.7 & 200 & 188 \\
\hline New Jersey & 165 & 235 & 156 \\
\hline
\end{tabular}

Table 5. Optimal values for DV and for OFs.

\begin{tabular}{|c|c|c|c|c|c|}
\hline $\begin{array}{c}\text { OPTIMAL } \\
\text { VALUES }\end{array}$ & $\mathrm{X} 1$ & $\mathrm{X} 2$ & $\mathrm{X} 3$ & $\begin{array}{c}\text { OF1 } \\
\text { (patients) }\end{array}$ & $\begin{array}{c}\text { OF2 } \\
\text { (MUS\$) }\end{array}$ \\
\hline Beds & 1,248 & 38,158 & 1,157 & 12,358 & 173.20 \\
\hline ICUs & 41 & 4,165 & 11 & 7,381 & 42.52 \\
\hline
\end{tabular}

Table 6. Optimal solution for patient transport problem analyzing medical resource 1 (hospital beds).

\begin{tabular}{|c|c|c|c|}
\hline Beds & Connecticut & Pennsylvania & Delaware \\
\hline New York & 1,248 & 17,044 & 82 \\
\hline New Jersey & 0 & 0 & 1,075 \\
\hline
\end{tabular}

Table 7. Optimal solution for patient transport problem analyzing medical resource 2 (ICUs).

\begin{tabular}{|c|c|c|c|}
\hline ICUs & Connecticut & Pennsylvania & Delaware \\
\hline New York & 414 & 4,165 & 0 \\
\hline New Jersey & 0 & 0 & 11 \\
\hline
\end{tabular}




\section{Cation for Figures:}

Figure 1: Classification of global search and optimization.

Figure 2: General optimization approach.

Figure 3: Patients distribution super structure.

Figure 4: Results after MNG for medical resource 1 (hospital beds).

Figure 5: Results after MNG for medical resource 2 (ICUs). 


\section{FIGURES}

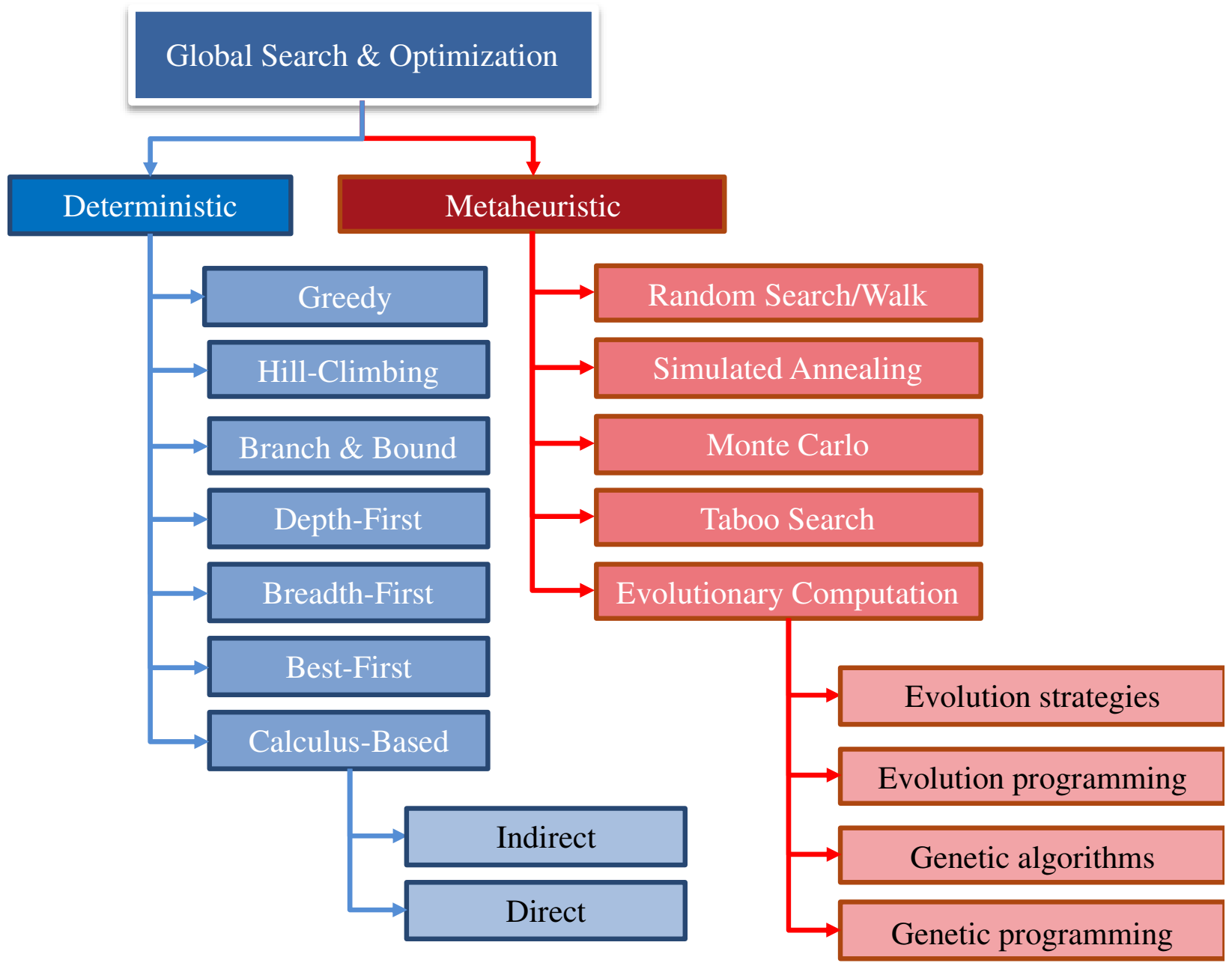

Figure 1. Classification of global search and optimization. 


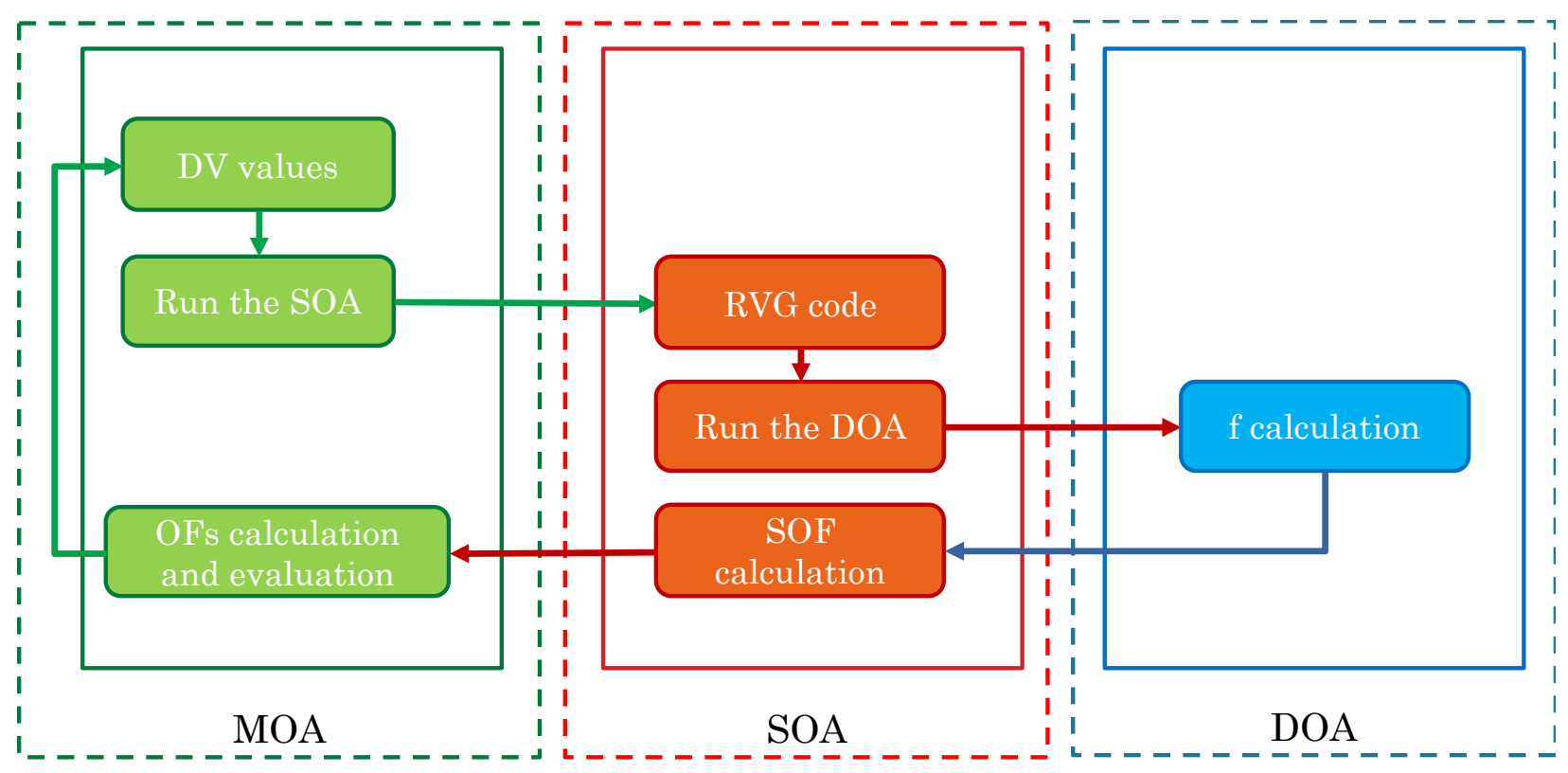

Figure 2. General optimization approach. 


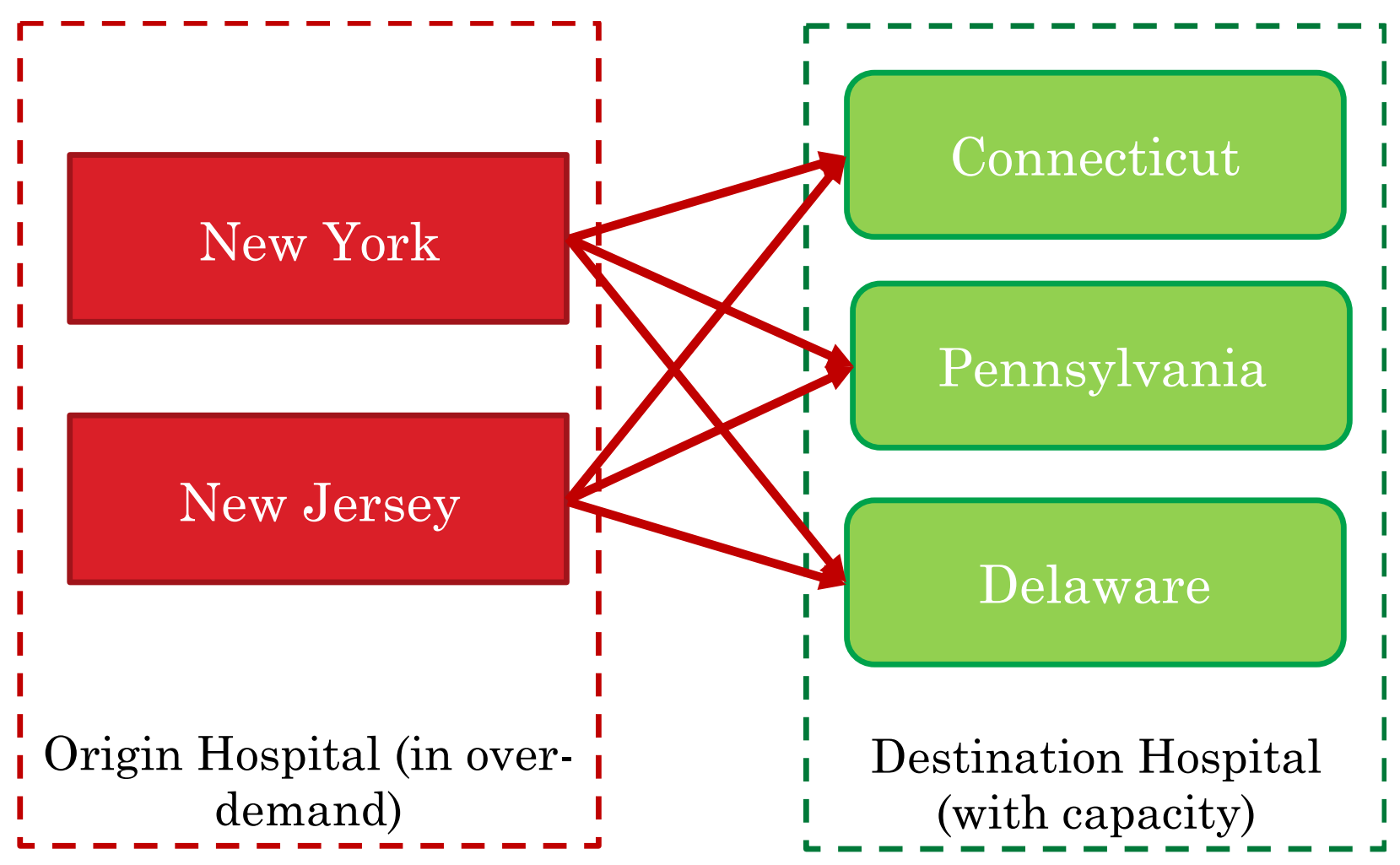

Figure 3. Patients distribution super structure. 


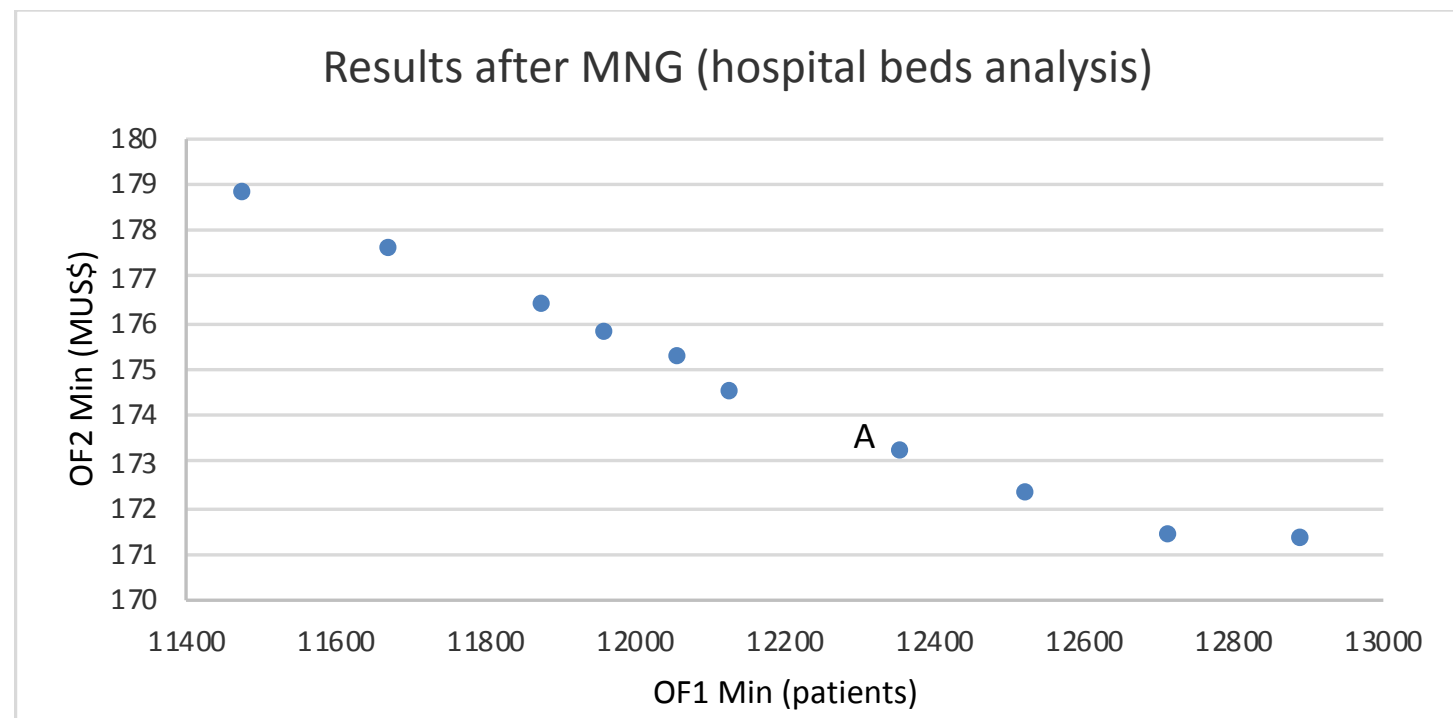

Figure 4. Results after MNG for medical resource 1 (hospital beds). 


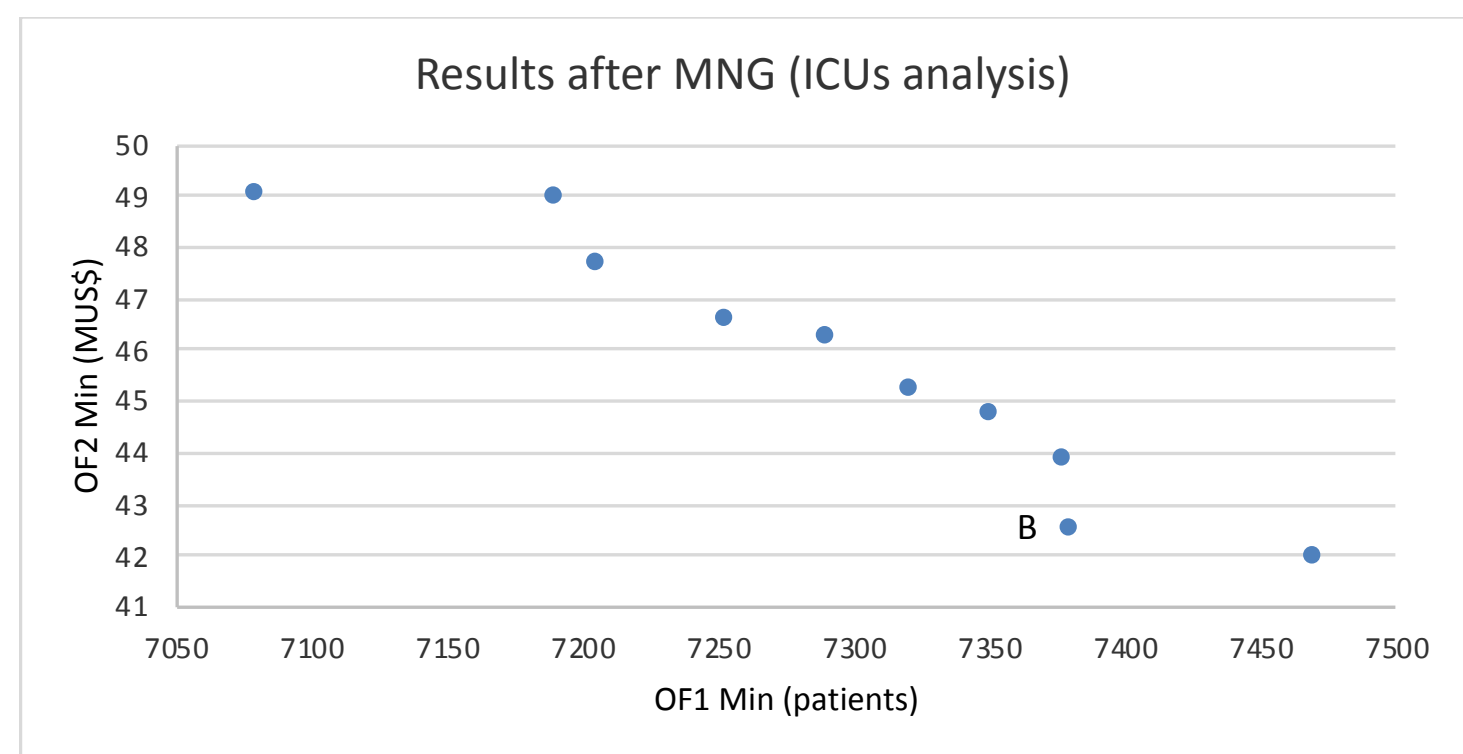

Figure 5. Results after MNG for medical resource 2 (ICUs). 


\section{Figures}

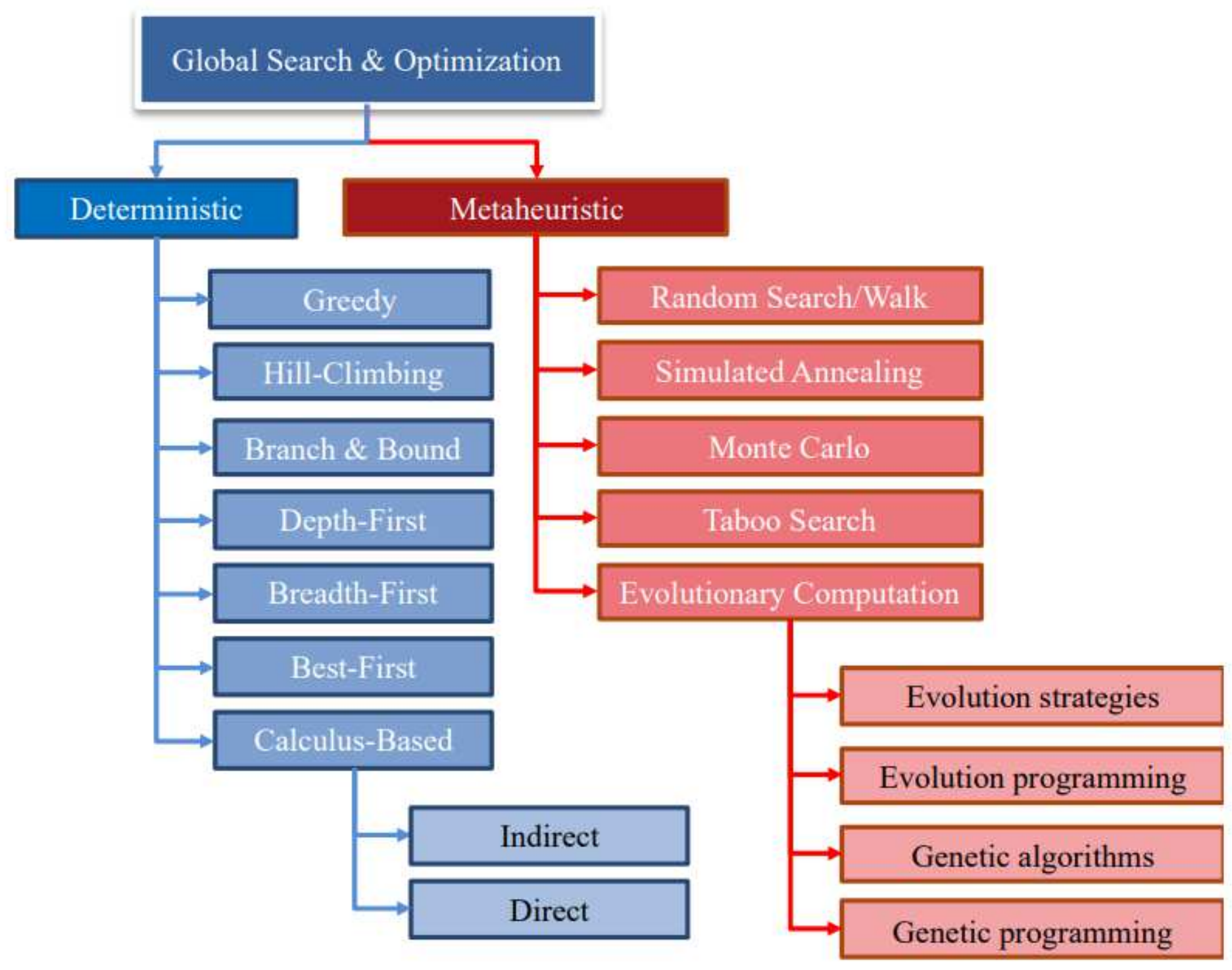

Figure 1

Classification of global search and optimization. 


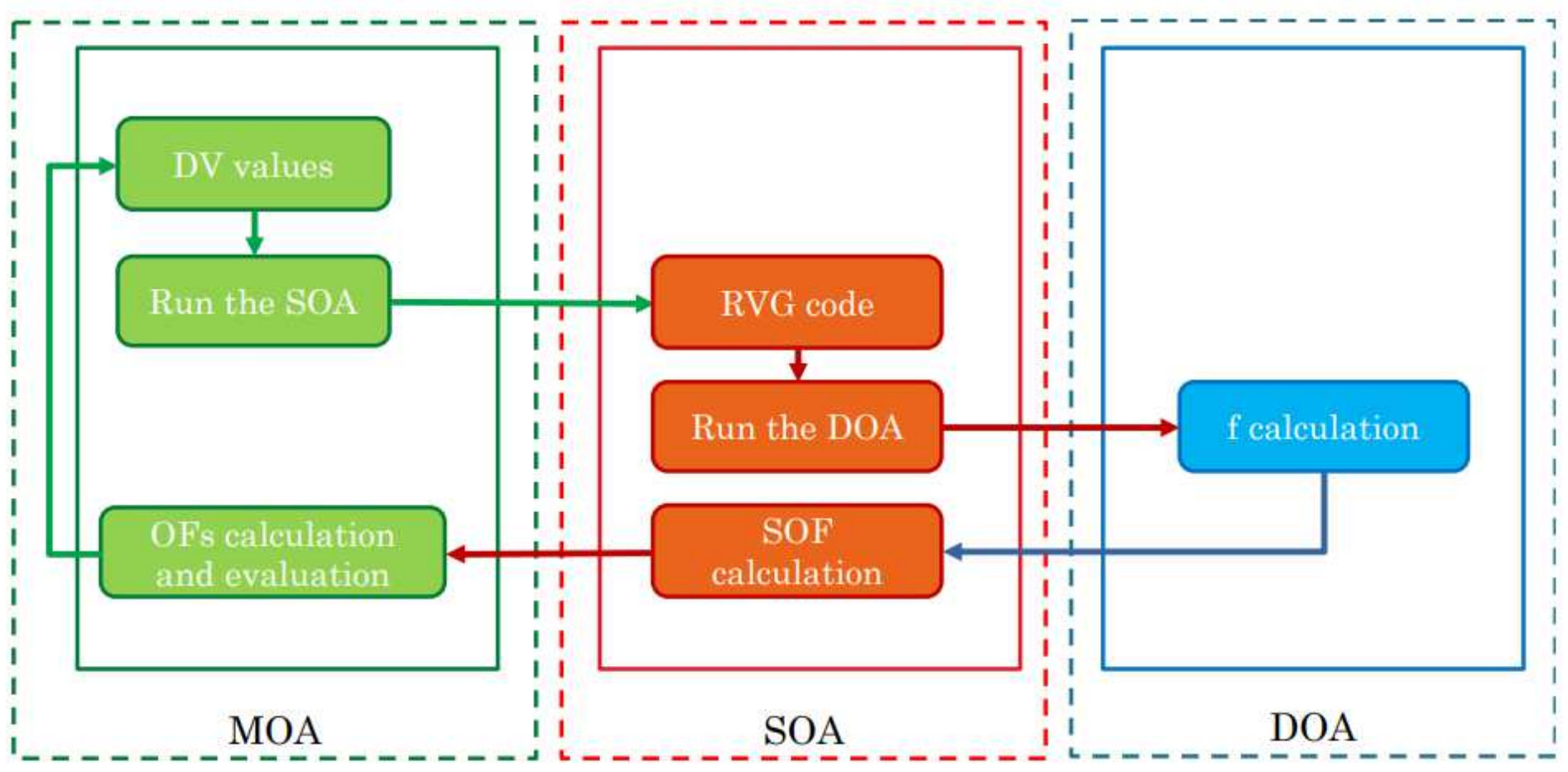

Figure 2

General optimization approach.

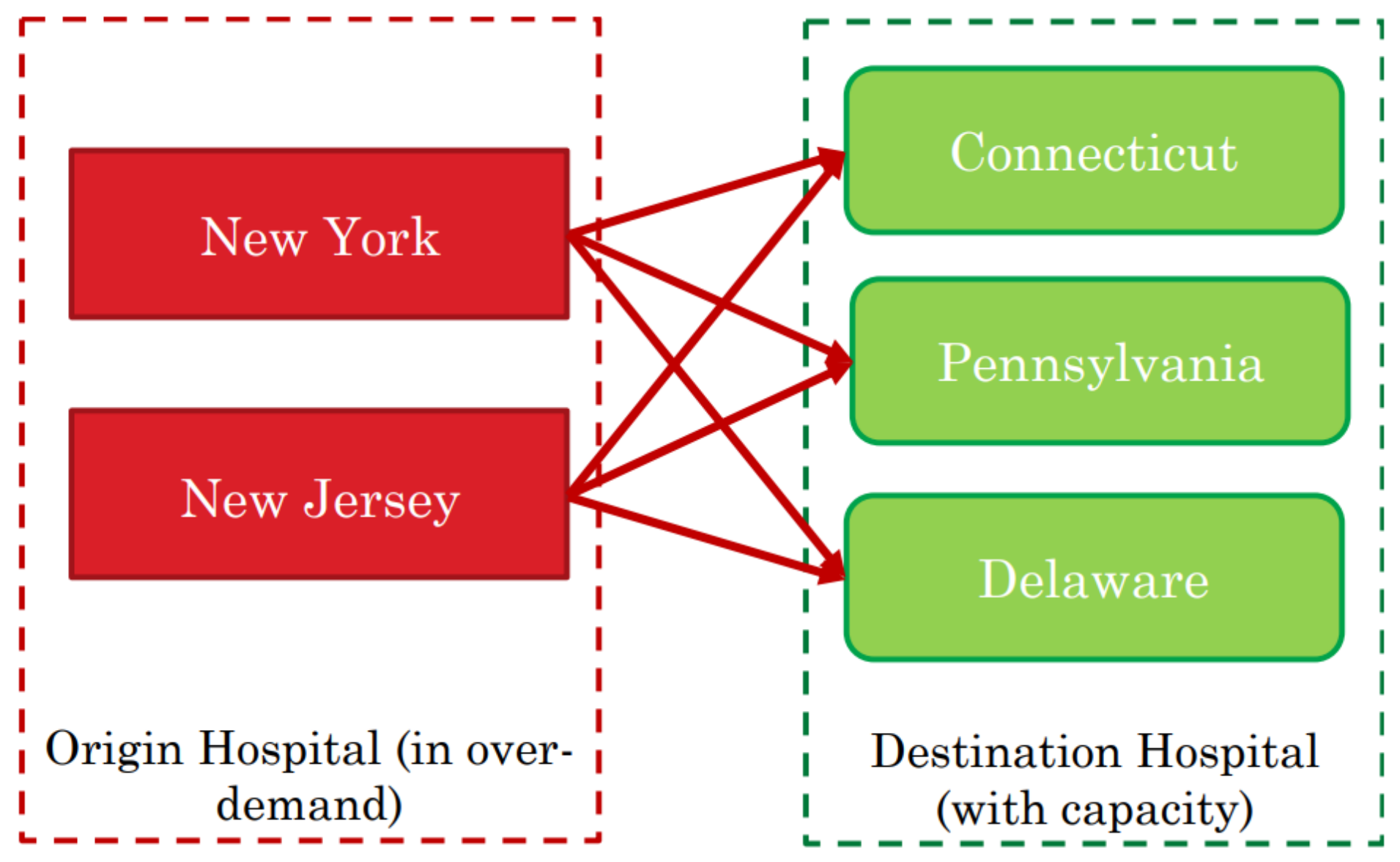

Figure 3 
Patients distribution super structure.

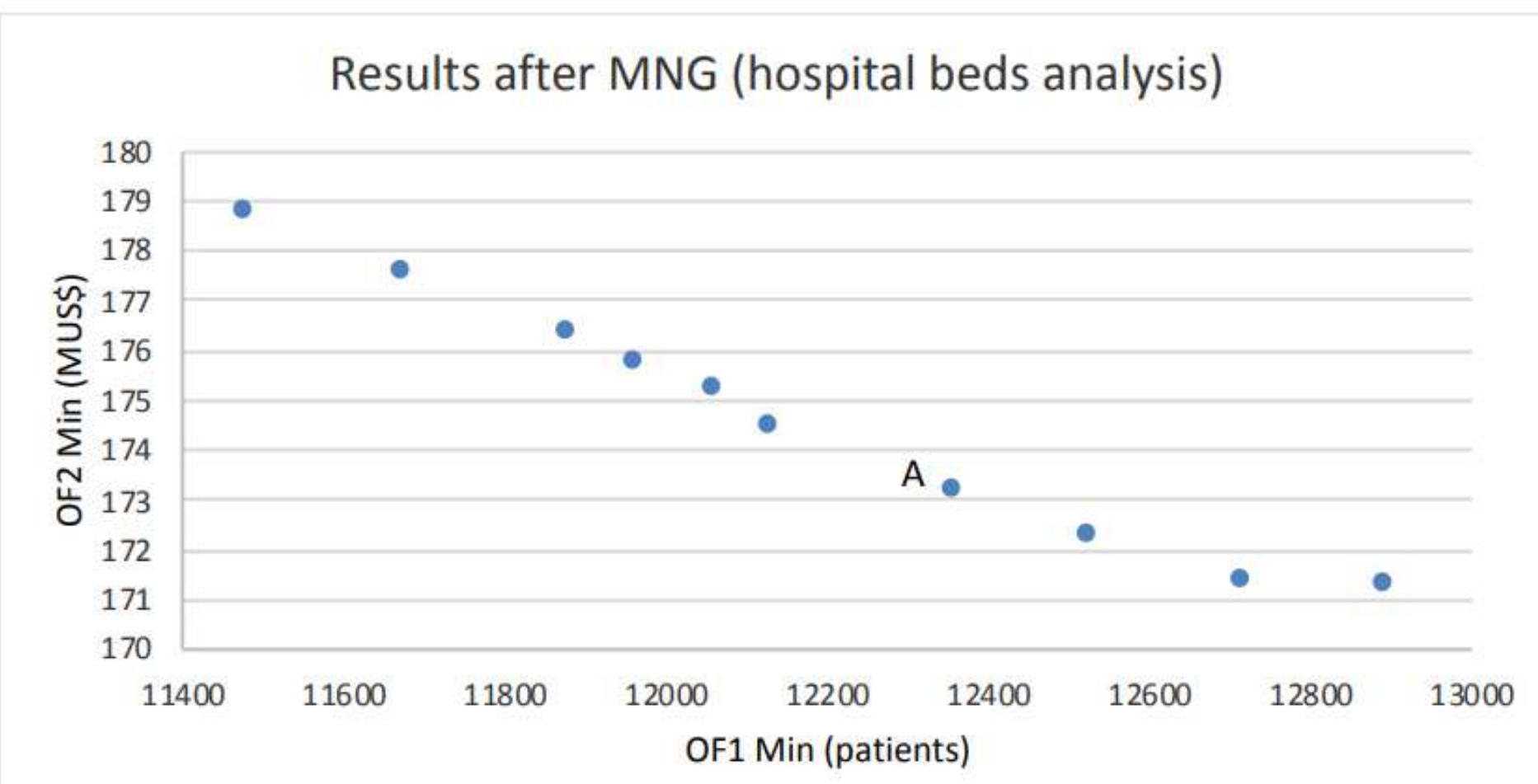

Figure 4

Results after MNG for medical resource 1 (hospital beds).

Results after MNG (ICUs analysis)

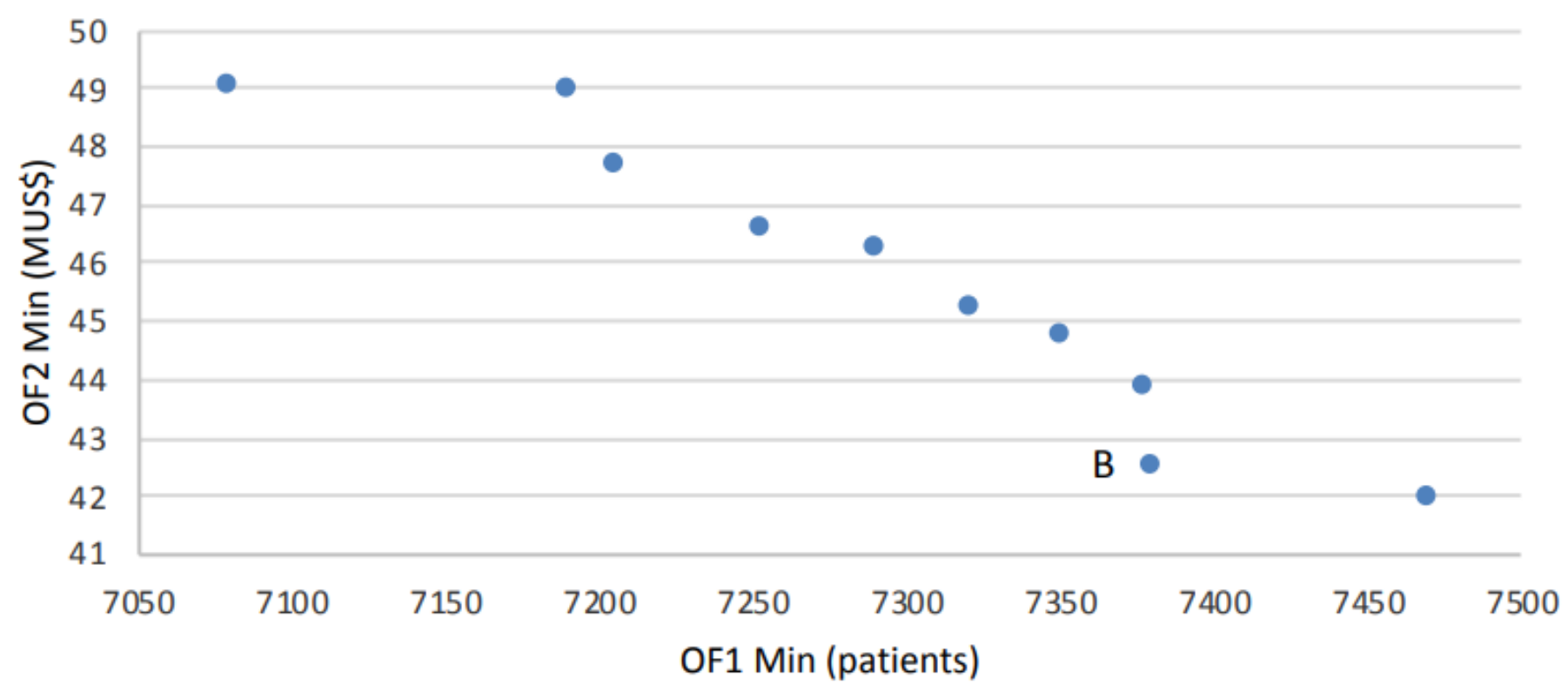

Figure 5 
Results after MNG for medical resource 2 (ICUs). 